\title{
UNA APROXIMACIÓN ESTILÍSTICO-TECNOLÓGICA A LA CERÁMICA POLÍCROMA 'VAQUERÍAS' DEL NOROESTE ARGENTINO
}

\author{
María Fabiana Bugliani y Lucas Pereyra Domingorena ${ }^{1}$
}

\begin{abstract}
Resumen
Bajo el rótulo 'Vaquerías' se conoce la primera cerámica polícroma del Noroeste Argentino, ampliamente dispersa pero siempre exigua y tradicionalmente definida como un bien de alto valor simbólico. Se presentan en esta oportunidad los resultados del análisis tecnológico-

estilístico sobre los materiales 'Vaquerías' procedentes de los sitios

Cardonal y Bordo Marcial (Catamarca, Argentina) y se evalúa su presencia dentro de un contexto doméstico aldeano del 100 DC. Esta nueva evidencia, resultado de los estudios arqueométricos integrados

al análisis del diseño, ha permitido ampliar el conocimiento de los objetos 'Vaquerías' y evaluar la producción, circulación y consumo de estos bienes como prácticas sociales significativas.
\end{abstract}

Palabras claves: petrografía - morfología - iconografía.

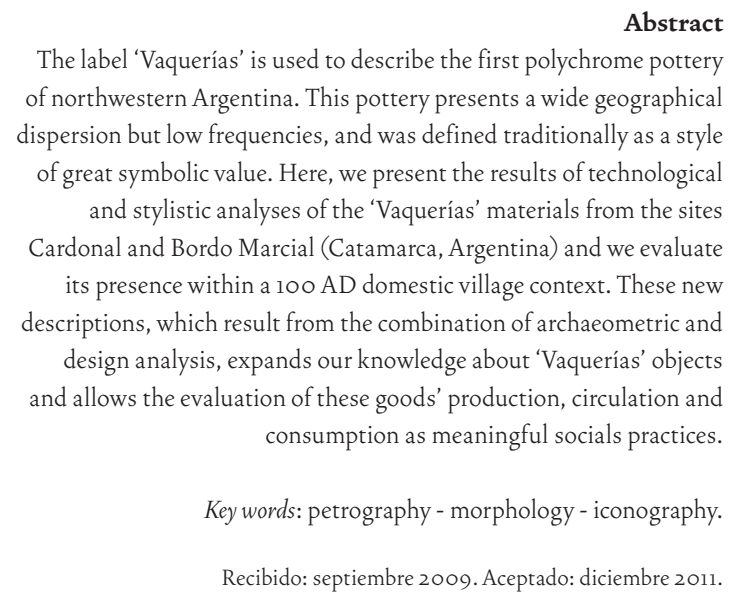

Abstract

The label 'Vaquerías' is used to describe the first polychrome pottery of northwestern Argentina. This pottery presents a wide geographical dispersion but low frequencies, and was defined traditionally as a style great symbolic value. Here, we present the results of technological and stylistic analyses of the 'Vaquerías' materials from the sites its presence within a $100 \mathrm{AD}$ domestic village context. These new escriptions, which result from the combination of archaeometric and design analysis, expands our knowledge about 'Vaquerías' objects consumption as meaningful socials practices.

Recibido: septiembre 2009. Aceptado: diciembre 2011.

${ }^{1}$ CONICET - Museo Etnográfico "Juan B. Ambrosetti", Facultad de Filosofía y Letras, Universidad de Buenos Aires. Moreno 350 (1091),

Buenos Aires, ARGENTINA. Email: fbugliani@gmail.com y lucasdomingorena@gmail.com

\section{* Introducción}

Con el rótulo 'Vaquerías', se conoce un estilo cerámico polícromo con predominio de diseños geométricos pintados y elaborada terminación, que ha sido localizado en sitios del período Formativo (600 AC-100o DC, según Núñez Regueiro 1974) en diferentes áreas del Noroeste Argentino (NOA).

Heredia y Pérez (1972) establecieron esta denominación a partir del examen de una serie de vasijas de colecciones y de la recopilación de datos obtenidos por otros investigadores en distintos sitios. La presencia de un conjunto cerámico diferenciable de lo conocido hasta el momento había sido previamente advertida por González, quien lo denominó Condorhuasi Tricolor (1956). Posteriormente Korstanje $(1995,1998)$ realizó un relevamiento exhaustivo de todas las evidencias publicadas hasta esa fecha y de las piezas Vaquerías presentes en diferentes colecciones privadas y de museos. A partir de la información disponible, realizó una caracterización del estilo considerando los aspectos decorativos y morfológicos. Asimismo, planteó interrogantes en torno a su origen, dispersión, asociaciones culturales y cronológicas.

En los primeros estudios, la cerámica Vaquerías fue calificada como material 'alóctono' o 'intrusivo' pero sin discutirse en profundidad las implicancias de su presencia en determinado contexto cultural o cronológico, ni se consideraron las asociaciones con el material 'local'. Asimismo, se planteó que Vaquerías jugaría "el papel de tresd'union entre las culturas del Este [...] con las manifestaciones 
tempranas del área valliserrana [...] y de la Puna..." (Heredia et al. 1974a: 150), cuestión que no ha sido posteriormente retomada. También se asumió que estas cerámicas eran bienes de alto valor simbólico y de uso ritual (González y Baldini 1989; González 1998) por encontrarse formando parte del ajuar de enterratorios, poseer una alta dispersión geográfica y una baja frecuencia relativa dentro de contextos arqueológicos excavados sistemáticamente.

Nuestro trabajo actualiza la información con que se cuenta a la fecha y aporta nuevos datos a partir del análisis de materiales Vaquerías de los sitios Cardonal y Bordo Marcial, dos contextos domésticos aldeanos del 100 DC ubicados en la localidad de La Quebrada (valle del Cajón, Catamarca, Argentina), en una zona que no había reportado hasta el momento una presencia significativa de este estilo. En esta oportunidad, se presenta una caracterización a partir de la evaluación del repertorio morfológico e iconográfico y del análisis petrográfico de las pastas cerámicas. El objetivo es integrar dichos análisis a fin de lograr una comprensión abarcadora de esta alfarería.

\section{* VAquerías y SU dispersión geográfica}

La cerámica Vaquerías ha sido detectada en diferentes sectores del NOA (Figura 1), ${ }^{2}$ llevando a plantear una amplia dispersión para este estilo (Heredia et al. 1974a; Korstanje 1995, 1998; Ortiz 2007). Se ha registrado en los valles de Hualfín, del Bolsón, Santa María, Calchaquí Norte, del Cajón, Tafí, Lerma, Quebrada del Toro, Puna y Yungas y se han encontrado también fragmentos en algunos sitios del norte de Chile (Tabla 1). A pesar de esta vasta distribución geográfica, la cerámica Vaquerías generalmente aparece representada por una escasa proporción de fragmentos en cada sitio.

Asimismo, si consideramos las piezas enteras conocidas pertenecientes a colecciones de museos o privadas (Figura 2), en total pueden contarse 52. De las mismas, 14 no poseen ningún dato de procedencia, y 31 presentan su origen indicado vagamente a través del nombre de la localidad, el valle o la provincia. Las siete restantes han

\footnotetext{
2 Algunos sitios no han podido ser localizados en el mapa dado que su ubicación geográfica no ha sido publicada con precisión.
}

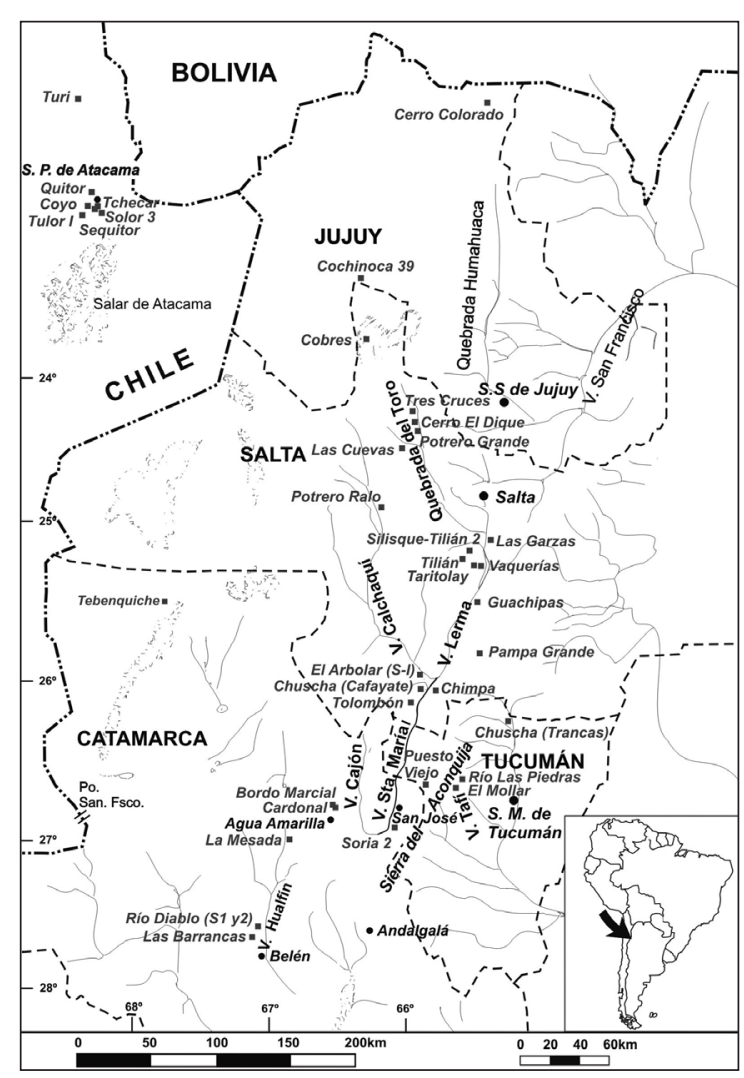

Figura 1. Sitios y localidades del Noroeste Argentino y norte de Chile mencionados en el texto con presencia de cerámica Vaquerías.

sido recuperadas en trabajos arqueológicos y fueron encontradas en contextos funerarios.

De nuestra área de estudio, el valle del Cajón, solamente se conoce una pieza completa Vaquerías (Figura 3a). Una vasija de perfil compuesto, cuya parte inferior es un elipsoide horizontal, de base plana pintada con motivos geométricos (rombos, triángulos, líneas rectas y quebradas) articuladas a través de reflexión especular y traslación horizontal (Korstanje 1998: 91).3 En este mismo valle, Scattolin y Gero, en los años 90, registraron fragmentos de estilo Vaquerías en sus prospecciones en la localidad de La Quebrada. En trabajos de

\footnotetext{
3 Esta pieza, procedente de la localidad de Agua Amarilla, se alojaba en el Instituto de Arqueología y Museo de la Universidad Nacional de Tucumán hasta que fue robada en abril de 1997 (Korstanje 1998).
} 


\begin{tabular}{|c|c|c|}
\hline Área & Sitio/Zona & Referencias Bibliográficas \\
\hline \multirow{3}{*}{ Valle de Hualfín } & Dpto. Belén (colecciones) & Heredia et al. 1974a \\
\hline & Sitio 1 y 2 del Río Diablo & González y Cowgill 1975 \\
\hline & Las Barrancas ${ }^{1}$ & Berberián y Massidda 1975 \\
\hline Bolsón de Andalgalá & Dpto Andalgalá (colección) & Heredia et al. $1974 \mathrm{a}$ \\
\hline Valle del Bolsón & La Mesada & Korstanje 1998 \\
\hline \multirow{3}{*}{ Valle de Tafí } & Río Las Piedras & Cremonte 1996 \\
\hline & El Mollar & Núñez Regueiro y García Azcárate 1996 \\
\hline & Puesto Viejo. Quebrada de Los Corrales & Gramajo Bühler 2009; Oliszewski et al. 2010 \\
\hline \multirow{3}{*}{ Valle del Cajón } & Agua Amarilla (colección) & Korstanje 1995, 1998 \\
\hline & Cardonal & Scattolin et al. 2007a, b \\
\hline & Bordo Marcial & Pereyra Domingorena 2009 \\
\hline \multirow{5}{*}{ Valle Santa María } & Tolombón² & \\
\hline & Chuscha $^{3}$ & Tarragó y Scattolin 1999 \\
\hline & Chimpa & Heredia et al. $1974^{\text {a }}$ \\
\hline & San José (colección) & Korstanje 1995,1998 \\
\hline & Soria 2 & Palamarczuk et al. 2007 \\
\hline \multirow{2}{*}{ Valle Calchaquí Norte } & Potrero Ralo & Tarragó 1996 \\
\hline & El Arbolar. Sitio $1^{4}$ & Tarragó y Scattolin 1999 \\
\hline Yungas tucumanas & Chuscha & Heredia et al. $1974^{a}$ \\
\hline Puna catamarqueña & Tebenquiche & Haber 1996 \\
\hline \multirow{4}{*}{ Quebrada del Toro } & Las Cuevas & Cigliano 1970; Cigliano et al. 1972, 1976 \\
\hline & Cerro El Dique & Cigliano et al. 1972,1976 \\
\hline & Potrero Grande & Cigliano et al.1972, 1976 \\
\hline & Tres Cruces & De Feo 2007 \\
\hline \multirow{10}{*}{ Valle de Lerma } & San Nicolás & Cigliano et al. 1972 \\
\hline & Tilián & Cigliano et al. 1972 \\
\hline & Corral de la Viña & Cigliano et al. 1972 \\
\hline & Guachipas & Cigliano et al. 1972; Heredia et al. 1974a \\
\hline & Valle de los Demonios & Cigliano et al. 1972 \\
\hline & Vaquerías & $\begin{array}{c}\text { Cigliano et al. 1972; Heredia et al. 1974a; Raffino et } \\
\text { al. 1982; González y Baldini 1989 }\end{array}$ \\
\hline & Las Garzas & Cremonte et al. 1987 \\
\hline & Silisque Tilián 2 & Escobar 1996, 2008 \\
\hline & Taritolay & Escobar 2008 \\
\hline & Pampa Grande & Heredia et al. 1974a \\
\hline Puna salteña & Cobres & Santoni y Buliubasich 1988 \\
\hline \multirow{2}{*}{ Puna jujeña } & Cochinoca 39 & Fernández Distel 1998 \\
\hline & Cerro Colorado & Krapovikas $1977^{5}$ \\
\hline Subregión Río Salado (Chile) & Localidad de Turi & Núñez A. et al. 1975; Castro et al. 1994 \\
\hline \multirow{4}{*}{ San Pedro de Atacama (Chile) } & Tulor 1 & Llagostera et al. 1984 \\
\hline & Tulor algarrobo, Tchecar, Solor 3, Tulor, Coyo & Heredia et al. 1974 \\
\hline & Coyo & Núñez A. et al. 1975 \\
\hline & Sequitor, Quitor, Solor 3 & Tarragó 1989 \\
\hline
\end{tabular}

Tabla 1. Sitios con presencia de cerámica Vaquerías publicados.

1 En la publicación, los autores catalogaron a estas piezas Condorhuasi Tricolor y Condorhuasi Rojo sobre Ante. Posteriormente, Korstanje (1995) consideró que estas piezas debían ser consideradas Vaquerías, opinión con la que coincidimos.

2 La expedición Lafón-Krapovickas de 1956 halló dos fragmentos Vaquerías (56-26 y 56-45), material que se encuentra depositado en el Museo Etnográfico "Juan B. Ambrosetti" de Buenos Aires. De acuerdo al Catálogo $\mathrm{N}^{\circ} 12$ del Depósito de Arqueología, el fragmento 56-26 procede de una recolección en "las cercanías de la casa de Llampa". El fragmento 56-45 procede de "las cercanías de la casa de Cruz, Trinchera IV Superficie".

3 Las autoras remiten a una pieza de la Colección Bravo relevada y registrada por M. T. Carrara en los años sesenta. De acuerdo a Scattolin (2006), se trata de la pieza $\mathrm{N}^{\circ} 138$ de dicha colección.

4 Las autoras señalan que en el manuscrito de Heredia et al. (1974b MS) se menciona la presencia de material cerámico Vaquerías en el Sitio I de El Arbolar ubicado en los alrededores de la localidad de San Carlos (Salta).

5 Este material corresponde a lo que Krapovikas denominó Tipo Cerro Colorado Polícromo. Posteriormente, otros investigadores han asociado el material publicado por Krapovickas con el estilo Vaquerías a partir de semejanzas estilísticas (Tarragó 1989; Korstanje 1998). 


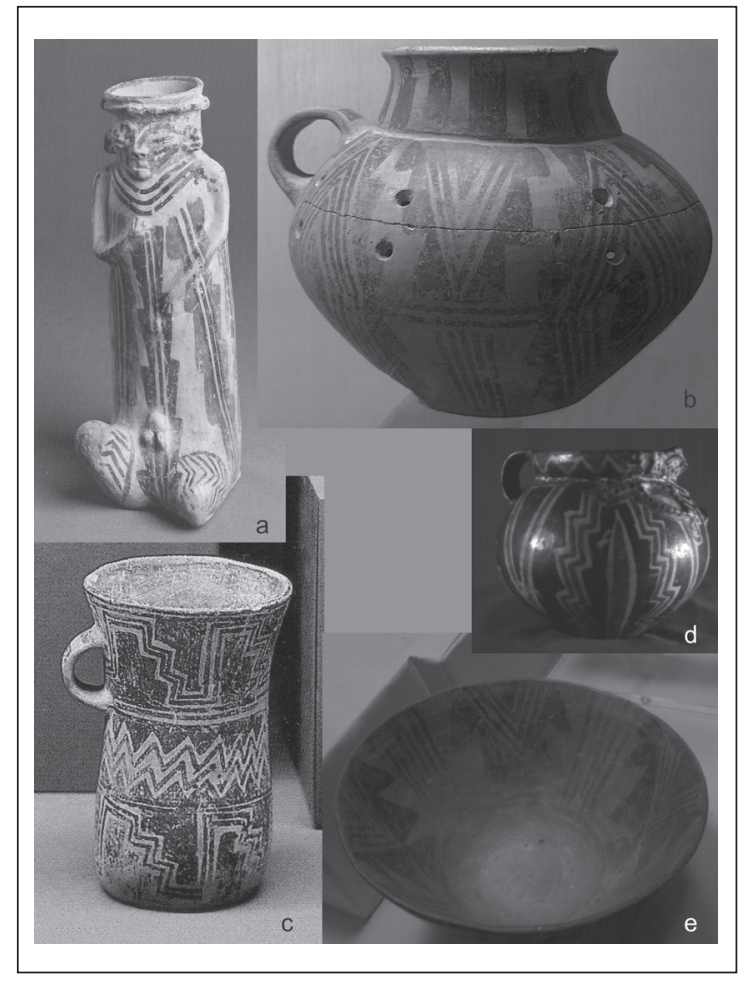

Figura 2. Piezas Vaquerías. a. Ministerio de Relaciones Exteriores, Comercio Internacional y Culto, Alto: $21,5 \mathrm{~cm}$; b. Museo Arqueológico Adán Quiroga de Catamarca, Alto: 20 $\mathrm{cm}$ aprox.; c. Ministerio de Relaciones Exteriores, Comercio Internacional y Culto, Alto: 16,4 cm.; d. Colección Zavaleta-Field Museum of Natural History of Chicago, Alto: $9 \mathrm{~cm}$.; e. Museo Etnográfico J. B. Ambrossetti, Alto: $8 \mathrm{~cm}$.

campo posteriores, se recuperaron materiales Vaquerías de contextos domésticos en los sitios Cardonal y Bordo Marcial.

Estos sitios corresponden a dos aldeas agrícolas datadas en los primeros siglos DC, ambas compuestas por cientos de recintos circulares y subcirculares, situados en una zona transicional entre la Puna y los valles mesotermales (Scattolin et al. 2007). Como en otras aldeas conocidas para esta época, el material cerámico recuperado consiste en una gran mayoría de fragmentos del grupo ordinario mientras que los materiales del grupo fino solamente alcanzan el $17,7 \%$ de la muestra e incluyen fragmentos grises muy pulidos, grises incisos con motivos geométricos, rojos pintados y algunos fragmentos con pequeños apliques modelados.

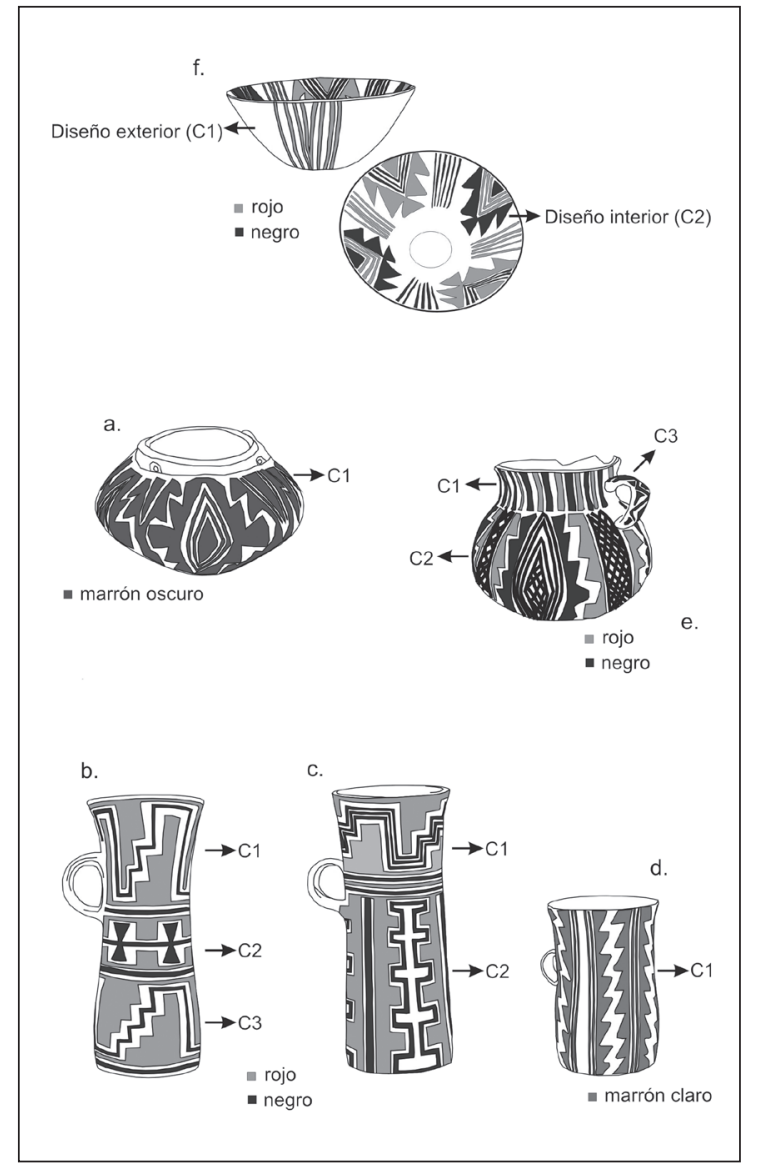

Figura 3. Diseños y campos de representación en la cerámica Vaquerías.

Dentro de este conjunto fino y entre los fragmentos que han recibido algún tratamiento plástico, se destacan los fragmentos del estilo Vaquerías pintados en diferentes tonalidades de rojos, negros y marrones sobre un engobe blanco amarillento (Figura 4a). El material fue hallado tanto en superficie como en los niveles estratigráficos excavados.

Dado que se trata de contextos domésticos bien recuperados y sistemáticamente estudiados, a diferencia de la mayoría de los datos publicados donde la presencia de cerámica Vaquerías es solo mencionada, nos parece relevante abordar el estudio de esta alfarería integrando diferentes aspectos del estilo -algunos de ellos aún no abordados como el petrográfico- y atendiendo a los contextos de circulación y uso concretos. 


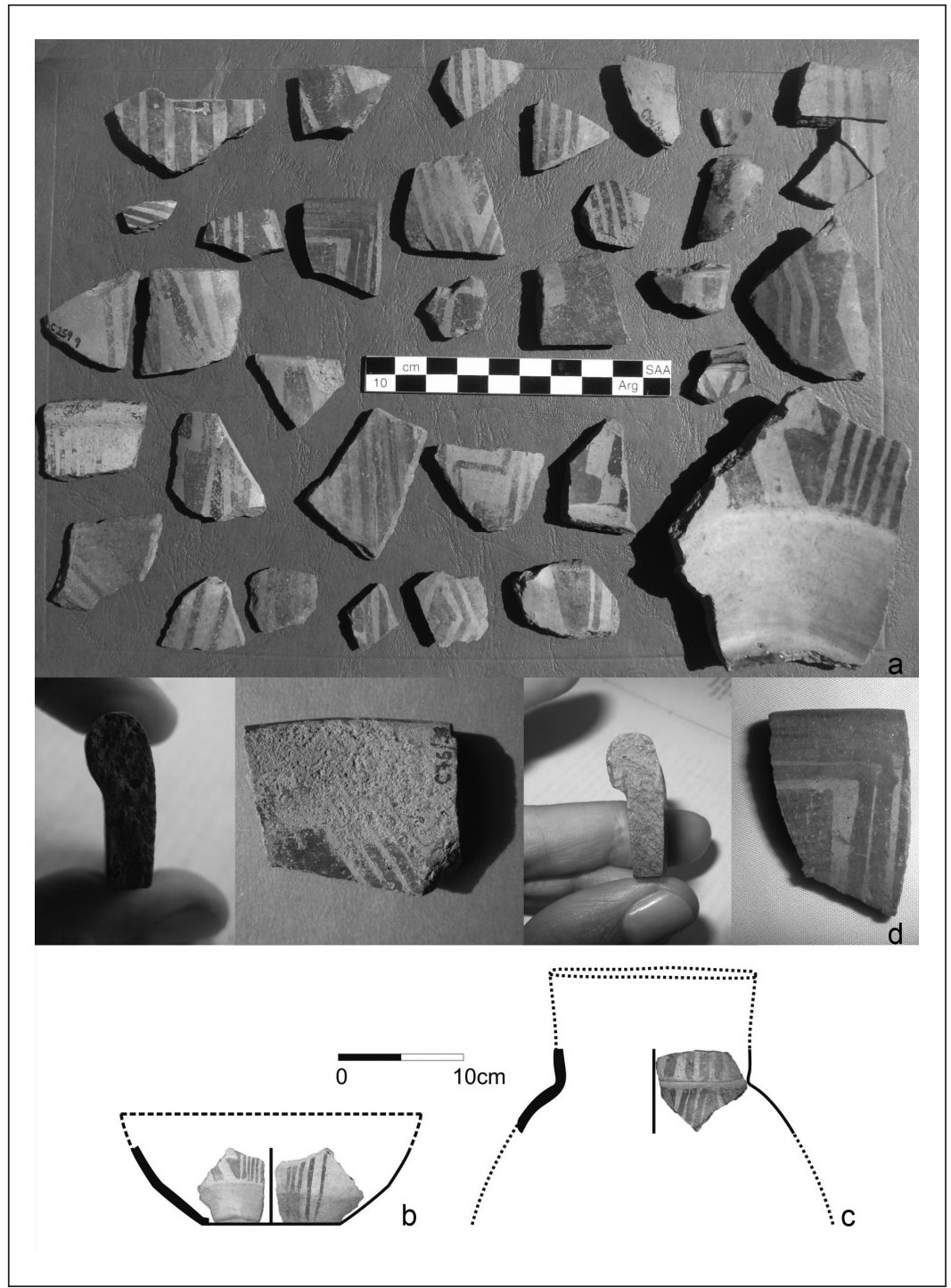

Figura 4. a. Fragmentos Vaquerías de Cardonal y Bordo Marcial, La Quebrada (valle del Cajón); b. Cuenco de perfil compuesto, Bordo Marcial; c. Jarra de cuerpo subglobular, Cardonal; d. Fragmentos con borde engrosado.

\section{* El diseño vaquerías: Formas y figuras}

Hasta el momento, el repertorio morfológico conocido para Vaquerías incluye unas pocas formas muy características que pueden presentarse con algunas variantes y otras de las cuales se conocen pocos ejemplos.

Una de las formas corresponde a vasos cilindricos o subcilindricos de paredes levemente cóncavas, no restringidos. Presentan bordes a veces evertidos con labio recto o con- vexo, base plana y asa vertical subrectangular (Figuras 2c, 3b, 3c y 3d). Otras son jarras de cuerpo subglobular, con cuello corto cilíndrico o de paredes levemente cóncavas (Figuras 2 b y 3 e). Presentan bordes evertidos, muchas veces engrosados y en la unión cuello-cuerpo se distingue un punto angular a veces resaltado con una excisión. Por último, en menor medida existen cuencos de perfil compuesto, con punto angular en el tercio inferior de la altura de la pieza, paredes suavemente cóncavas y borde por lo general engrosado (Figura 4b). 
También se han detectado otras formas menos frecuentes y estandarizadas, tales como cuencos hemisféricos de perfil simple (Figuras ze y $3 \mathrm{f}$ ), vasijas subglobulares de base plana y de cuello corto (Figura $2 \mathrm{~d}$ ) y vasijas ovoides de cuello corto.

En la muestra de Cardonal y Bordo Marcial, el estado de fragmentación impide, en la mayoría de los casos, conocer la forma completa del recipiente a pesar de lo cual pudieron identificarse tres cuencos y una jarra de cuerpo subglobular (Figuras 4 b y 4c). También aparecen los bordes engrosados que fueran mencionados como característicos de este estilo (Figura $4 \mathrm{~d}$ ) y que se registraron en otros lugares como Chimpa, Chuscha, Guachipas (Heredia et al. 1974a) y Tolombón (Tarragó y Scattolin 1999). Asimismo, se observan otros tipos como los bordes directos, levemente evertidos y de labio plano que también fueron hallados en piezas Vaquerías de Quebrada del Toro (Cigliano et al. 1972) y del valle de Hualfín (Heredia et al. 1974a).

Las representaciones visuales de este estilo son predominantemente geométricas (ver Figura 3). Sin embargo, como ya lo han resaltado otros autores, existen ejemplos de figuración en varias de las piezas enteras conocidas, donde pueden distinguirse figuras humanas y animales logradas a través del modelado de toda o gran parte de la vasija (González y Baldini 1989; Korstanje 1998) (Figura 2a). Otras veces, la representación de personajes o animales fue lograda a través de pequeños apéndices modelados. Más allá de estas representaciones figurativas, en todos los casos están presentes los motivos geométricos característicos del estilo completando o complementando el diseño figurativo.

En la muestra de Cardonal y Bordo Marcial, los diseños son únicamente geométricos, registrándose figuras sólidas y figuras de línea. Entre las figuras sólidas aparecen triángulos, rombos escaleriformes y triángulos unidos por unos de sus vértices (a veces denominados banderines). Las figuras de línea están presentes como líneas quebradas múltiples en forma de $\mathrm{V}$, líneas quebradas en forma escalonada y líneas paralelas verticales. Las configuraciones mayores se logran a partir de la combinación de ambos tipos de elementos. La mayoría de las veces las figuras rellenas están orladas por figuras de líneas.
Los campos de representación para estos diseños geométricos guardan estrecha relación con la forma de la vasija. Los cuencos suelen presentar diseños pintados tanto en su interior como en su lado externo, aunque puede variar la sección elegida en cada caso (Figura 3f). En el interior de los cuencos de perfil compuesto, el sector donde se ubican las representaciones está claramente delimitado por el punto angular que define la silueta. Los diseños geométricos se ubican en el segmento superior entre el borde y el punto angular. En cambio, en la cara externa de estas piezas, los diseños suelen extenderse tanto en el segmento superior del cuerpo como en el inferior. Otro elemento característico del diseño de estos cuencos es que los bordes, casi siempre engrosados o salientes, se hallan pintados dando un delineado, generalmente oscuro, a la terminación de la pieza.

Los vasos cilíndricos y subcilíndricos suelen estar pintados con diseños geométricos en toda la extensión de su cuerpo. En algunos casos, estas representaciones se extienden de borde a base en un solo campo (Figura 3d). En otras, el cuerpo está segmentado en dos o tres campos o paneles con diseños diferentes (FiguraS 3 b y $3 c$ ). Esta segmentación suele delimitarse a nivel de la inflexión de la pieza -si es que existe- o está marcada por una línea pintada que separa ambos diseños.

Por su parte, las jarras de contornos inflexionados o compuestos también presentan dos campos diferenciados con diseños distintos demarcados por el punto angular o de inflexión. Cuando las vasijas presentan asas, como ocurre en jarros y jarras, las mismas conforman otro campo decorativo con un diseño más simple (Figura 3e).

El color es uno de los elementos importantes en la cerámica Vaquerías, no solo porque se trata de un estilo pintado sino porque son los colores junto con los elementos geométricos seleccionados los que definen la configuración resultante. Si bien se habla de policromía, no todos los recipientes de este estilo tienen más de dos colores en sus diseños. Los autores que han descrito este material mencionan la presencia del negro, el rojo y el crema/blanco amarillento; sin embargo, hay que tener en cuenta que la policromía también está dada por la amplia variedad de tonalidades y las diferentes combinaciones que se registran en las piezas. En el caso de los materiales procedentes de Cardonal y Bordo Marcial, predominan los rojos, marro- 


\begin{tabular}{|c|c|c|c|c|c|c|}
\hline M & Fragmento & Figura & Forma & Campo Decorativo & Motivos & Colores utilizados \\
\hline 1 & $\mathrm{C}_{250-3}\left(\mathrm{~V}_{15}\right)$ & $5 \mathrm{a}$ & ¿Cerrada? & Superficie externa & $\begin{array}{c}\text { Rombos y triángulos en } \\
\text { hileras }\end{array}$ & $\begin{array}{c}\text { Marrones sobre fondo blanco } \\
\text { grisáceo }\end{array}$ \\
\hline 2 & $\mathrm{C}_{2} 6-\mathrm{T}_{45}\left(\mathrm{~V}_{9}\right)$ & $5 b$ & $\begin{array}{c}\text { Abierta. Cuenco de borde } \\
\text { engrosado }\end{array}$ & Superficie interna y externa & Rectángulos y líneas & $\begin{array}{l}\text { Rojo y negro sobre fondo } \\
\text { blanco }\end{array}$ \\
\hline 3 & $\mathrm{C}_{4} 4-\mathrm{T}_{3}(\mathrm{~V} 10)$ & $5 \mathrm{c}$ & ¿Abierta? & Superficie externa & Triángulos & Marrón sobre pasta natural \\
\hline 4 & $\mathrm{C}_{2} \mathrm{O} 6-\mathrm{T}_{2}\left(\mathrm{~V}_{19}\right)$ & $5 \mathrm{~d}$ & ¿Cerrada? & Superficie externa & Triángulos & $\begin{array}{c}\text { Negro y rojo sobre pasta } \\
\text { natural }\end{array}$ \\
\hline 5 & $\mathrm{C}_{1} 61-1\left(\mathrm{~V}_{2} \mathrm{O}\right)$ & $6 a$ & $\begin{array}{l}\text { Abierta. Cuenco de perfil } \\
\text { compuesto }\end{array}$ & Superficie interna y externa & $\begin{array}{c}\text { Triángulos y líneas } \\
\text { paralelas }\end{array}$ & $\begin{array}{c}\text { Marrones sobre fondo blanco } \\
\text { grisáceo }\end{array}$ \\
\hline 6 & $\mathrm{C}_{217-1}\left(\mathrm{~V}_{22}\right)$ & $6 b$ & ¿Cerrada? & Superficie externa & Triángulos en hilera & $\begin{array}{c}\text { Rojo y negro sobre pasta } \\
\text { natural }\end{array}$ \\
\hline 7 & $\mathrm{C}_{217-5}\left(\mathrm{~V}_{23}\right)$ & $6 c$ & ¿Cerrada? & Superficie externa & Líneas paralelas & Rojo sobre fondo crema \\
\hline 8 & $56-45$ & $6 \mathrm{~d}$ & Abierta & Superficie interna y externa & $\begin{array}{l}\text { Líneas paralelas y } \\
\text { triángulos }\end{array}$ & $\begin{array}{c}\text { Negro y rojo sobre pasta } \\
\text { natural }\end{array}$ \\
\hline
\end{tabular}

Tabla 2. Muestra de fragmentos analizados por petrografía.

nes, negro y un beige claro o blanco amarillento, existiendo tonos, valores de color y grados de saturación en una amplia escala. ${ }^{4}$ En la mayoría de los casos, se combinan diseños rojos y negros sobre una base blanco amarillento; en otros se utilizan dos tonalidades de marrones sobre esa misma base; el resto de los fragmentos son bicolores.

\section{* La pasta vaquerías: composición Y TECNOLOGÍA}

Como menciona Korstanje (1995, 1998), la mayoría de las descripciones de las pastas Vaquerías se han realizado a nivel macroscópico. Hasta el momento, se cuenta con una única descripción petrográfica publicada, correspondiente a un fragmento hallado en la quebrada de La Ciénega (Cremonte 1996). Por lo tanto, los resultados de los estudios petrográficos que aquí se exponen aportan un conocimiento distinto y a la vez más específico en relación a la composición de las pastas y a rasgos de su tecnología de producción.

4 El color fue determinado usando Tabla Munsell (1975, 2000). Los colores claros del engobe -crema, blanco amarillento- corresponden a 7.5YR 7/4, 7.5YR 6/4, 7.5YR 3/1, 7.5YR 5/4, 10YR $7 / 3$ y 5 YR $8 / 1$. Los rojos corresponden a $10 \mathrm{R} 3 / 3,10 \mathrm{R} 3 / 4,10 \mathrm{R}$ 3/6, $5 \mathrm{R}_{3} / 1,5 \mathrm{R}_{3} / 3,5 \mathrm{R}_{3} / 4,5 \mathrm{R}_{2} .5 / 3,7.5 \mathrm{R}_{4} / 4,7.5 \mathrm{R}_{3} / 4,7.5 \mathrm{R}_{3} / 6$, los marrones a $7.5 \mathrm{R}_{3} / 2,10 \mathrm{R}_{4} / 1,10 \mathrm{R}_{2} .5 / 1,10 \mathrm{R}_{2} .5 / 2,10 \mathrm{R}_{3} / 2$, 10YR 3/1, $5 \mathrm{YR}_{3} / 2,5 \mathrm{YR}_{3} / 4,5 \mathrm{YR}_{4} / 4$ y los negros a $2.5 \mathrm{YR} 2.5 / 1$, 5YR 2.5/1, 7.5YR 2.5/1.
Como se mencionó más arriba, la presencia de material Vaquerías en los sitios arqueológicos es exigua. En algunos casos se menciona la presencia de unos pocos fragmentos (Llagostera et al. 1984; Cremonte 1996; Palamarczuk et al. 2007), en otros se remarca la baja frecuencia de este conjunto en relación a los demás tipos cerámicos presentes en los contextos de excavación ( $\mathrm{Ci}$ gliano et al. 1972, 1976; Heredia et al. 1974a).

Los hallazgos Vaquerías en las aldeas de Cardonal y Bordo Marcial siguen este mismo patrón. Hasta el momento, se recuperaron 35 fragmentos, algunos de ellos procedentes de unidades de excavación y otros de recolecciones superficiales. Para el presente trabajo, se seleccionaron siete fragmentos, ${ }^{5}$ que corresponden al $20 \%$ de los tiestos Vaquerías. Éstos pertenecen a siete recipientes cerámicos distintos y expresan la variabilidad estilística y morfológica de la muestra recuperada (Tabla 2).

5 La muestra corresponde a los fragmentos: $\mathrm{C}_{250-3}$ (V15) hallado en Estructura 1 Unidad 4 Nivel superficie $(8 \mathrm{~cm}-35 \mathrm{~cm} \downarrow)$ Cardonal; C26-T45 (V9) hallado en Estructura 2 Unidad 3 Nivel 4 (75 cm - $85 \mathrm{~cm} \downarrow$ ) Cuadrícula C-Cardonal; C84-T3 (V10) hallado en Estructura 3 Unidad i Nivel $2(50 \mathrm{~cm}-60 \mathrm{~cm} \downarrow)$ Cuadrícula D-Cardonal; C206-T2 (V19) hallado en Estructura 18 Cuadrante NE Nivel 1 (o cm - $70 \mathrm{~cm} \downarrow)$ Bordo Marcial. En tanto que los fragmentos C161-1 (V20), C217-1 (V22) y C217-5 (V23) fueron hallados en recolecciones superficiales efectuadas en el sitio Bordo Marcial. 


\begin{tabular}{|c|c|c|c|c|c|c|c|c|}
\hline \multirow[b]{2}{*}{ M } & \multirow[b]{2}{*}{ Fragmento } & \multirow[b]{2}{*}{ Figura } & \multirow{2}{*}{$\begin{array}{l}\text { Estructura de } \\
\text { fondo }\end{array}$} & \multicolumn{3}{|c|}{ Inclusiones } & \multicolumn{2}{|c|}{ Cavidades } \\
\hline & & & & $\%$ & $\begin{array}{c}\text { Tipo } \\
\text { predominante }\end{array}$ & Granulometría predominante & Forma & $\begin{array}{c}\text { Tamaño } \\
\text { predominante }\end{array}$ \\
\hline 1 & $\mathrm{C}_{25} 50-3\left(\mathrm{~V}_{15}\right)$ & $5 \mathrm{a}$ & $\begin{array}{c}\text { Criptofilitosa algo } \\
\text { microgranosa }\end{array}$ & 24 & $\mathrm{P}-\mathrm{F}, \mathrm{A}, \mathrm{Qz}$ & $\begin{array}{c}\text { Arena gruesa } \\
(500 \mu \mathrm{m}-1000 \mu \mathrm{m})\end{array}$ & $\begin{array}{l}\text { Alargadase } \\
\text { irregulares }\end{array}$ & $400 \mu \mathrm{m}$ \\
\hline 2 & $\mathrm{C}_{2} 6-\mathrm{T}_{45}\left(\mathrm{~V}_{9}\right)$ & $5 b$ & Criptofilitosa & 21 & P-F, A, Qz & $\begin{array}{l}\text { Arena gruesa y arena muy gruesa } \\
\quad(500 \mu \mathrm{m}-2000 \mu \mathrm{m})\end{array}$ & $\begin{array}{l}\text { Alargadas e } \\
\text { irregulares }\end{array}$ & $400 \mu \mathrm{m}$ \\
\hline 3 & $\mathrm{C}_{4} 4-\mathrm{T}_{3}\left(\mathrm{~V}_{10}\right)$ & $5 \mathrm{c}$ & Criptofilitosa & 25 & P-F, A, Qz, TM & $\begin{array}{l}\text { Arena gruesa y arena muy gruesa } \\
\qquad(500 \mu \mathrm{m}-2000 \mu \mathrm{m})\end{array}$ & $\begin{array}{l}\text { Alargadase } \\
\text { irregulares }\end{array}$ & $500 \mu \mathrm{m}$ \\
\hline 4 & $\mathrm{C}_{2} 06-\mathrm{T}_{2}\left(\mathrm{~V}_{19}\right)$ & $5 \mathrm{~d}$ & $\begin{array}{c}\text { Criptofilitosa algo } \\
\text { microgranosa }\end{array}$ & 23 & Qz, P-F, A, TM & $\begin{array}{l}\text { Arena y arena gruesa } \\
(250 \mu \mathrm{m}-1000 \mu \mathrm{m})\end{array}$ & $\begin{array}{l}\text { Alargadas e } \\
\text { irregulares }\end{array}$ & $400 \mu \mathrm{m}$ \\
\hline 5 & $\mathrm{C}_{161-1}\left(\mathrm{~V}_{20} \mathrm{O}\right)$ & $6 a$ & $\begin{array}{l}\text { Criptofilitosa algo } \\
\text { microgranosa }\end{array}$ & 23 & P-F, A, Qz & $\begin{array}{l}\text { Arena gruesa y arena muy gruesa } \\
\quad(500 \mu \mathrm{m}-2000 \mu \mathrm{m})\end{array}$ & $\begin{array}{l}\text { Alargadase } \\
\text { irregulares }\end{array}$ & $400 \mu \mathrm{m}$ \\
\hline 6 & $\mathrm{C}_{217-1}\left(\mathrm{~V}_{22}\right)$ & $6 b$ & $\begin{array}{l}\text { Criptofilitosa algo } \\
\text { microgranosa }\end{array}$ & 18 & P-F, Qz, TM & $\begin{array}{c}\text { Arena y arena gruesa }(250 \mu \mathrm{m}- \\
1000 \mu \mathrm{m})\end{array}$ & $\begin{array}{l}\text { Alargadase } \\
\text { irregulares }\end{array}$ & $400 \mathrm{ctm}$ \\
\hline 7 & $\mathrm{C}_{217-5}\left(\mathrm{~V}_{23}\right)$ & $6 c$ & $\begin{array}{c}\text { Criptofilitosa algo } \\
\text { microgranosa }\end{array}$ & 26 & TM, P-F, A, Qz & $\begin{array}{l}\text { Arena gruesa y arena muy gruesa } \\
\qquad(500 \mu \mathrm{m} \text { a } 2000 \mu \mathrm{m})\end{array}$ & $\begin{array}{l}\text { Alargadas e } \\
\text { irregulares }\end{array}$ & $400 \mu \mathrm{m}$ \\
\hline 8 & $56-45$ & $6 \mathrm{~d}$ & Criptofilitosa & 25 & P-F, A, TM, Qz & $\begin{array}{c}\text { Arena gruesa } \\
(500 \mu \mathrm{m}-1000 \mu \mathrm{m})\end{array}$ & $\begin{array}{l}\text { Alargadas, } \\
\text { irregulares y } \\
\text { redondeadas }\end{array}$ & $400 \mu \mathrm{m}$ \\
\hline
\end{tabular}

Tabla 3. Síntesis del análisis petrográfico. P-F: pizarra-filita; Qz: cuarzo; A: arenisca; TM: tiesto molido.

Comparativamente, se analizó un fragmento Vaquerías (56-45) recuperado por Lafón y Krapovickas en las cercanías de la localidad de Tolombón durante los trabajos arqueológicos realizados en el año 1956. Las cerámicas por ellos recolectadas, asociables a los primeros siglos de la era cristiana, resultan de interés por dos motivos: 1) representan la cultura material de las sociedades anteriores al siglo IX DC, en un área en donde se privilegiaron los estudios sobre el período Tardío (1000 DC-1450 DC, ver Tarragó y Scattolin 1999; Scattolin 2000); y 2) provienen del norte del valle de Santa María, el cual debió ser un espacio nodal en las comunicaciones e intercambio de las antiguas poblaciones que habitaron este valle, el Calchaquí Norte y la quebrada de Las Conchas.

El análisis petrográfico ${ }^{6}$ se efectuó con un microscopio de polarización Leica DM EP. La descripción de los cortes delgados consistió, en primer lugar, en la caracterización del fondo de pasta, teniendo en cuenta el color y los microcristales observados, así como la textura de la matriz arcillosa. En segundo lugar, se identificaron las inclusio-

\footnotetext{
6 Para la identificación mineralógica se utilizó como bibliografía de referencia Mineralogía óptica de Paul F. Kerr (1965).
}

nes mayores a $15 \mu \mathrm{m}$, clasificándolas en cristaloclastos, litoclastos, minerales opacos, vidrio volcánico, gránulos de arcilla y tiesto molido. Esta identificación petrográfica se basó en las propiedades ópticas que cada uno de los minerales presentan: relieve, birrefringencia, pleocroísmo, ángulos de extinción, etc. Las inclusiones fueron clasificadas según la granulometría registrada, utilizando la tabla de Wentworth (publicada en Adams et al. 1997 [1984]). Por último, se describieron las cavidades, teniendo en cuenta las formas y tamaños observados. Una vez completado el proceso descriptivo, se realizó un conteo de un mínimo de 300 puntos (point counter) para poder obtener la distribución modal de la matriz, las inclusiones y las cavidades.

Los cortes delgados de las muestras analizadas presentan en general una matriz de color castaño, en algunos casos rojizo ( $\left.\mathrm{M}_{3}, \mathrm{M}_{7}, \mathrm{M} 8\right)$ y en otros (M2) castaño oscu-

\footnotetext{
7 En el caso de las pastas Vaquerías, el tiesto molido presenta la misma composición que las propias pastas, pero se puede reconocerlo porque las inclusiones al interior del mismo tienen orientaciones diferentes a las inclusiones no plásticas registradas en la matriz y una coloración más negruzca producto de la sobrecocción de los mismos.
} 


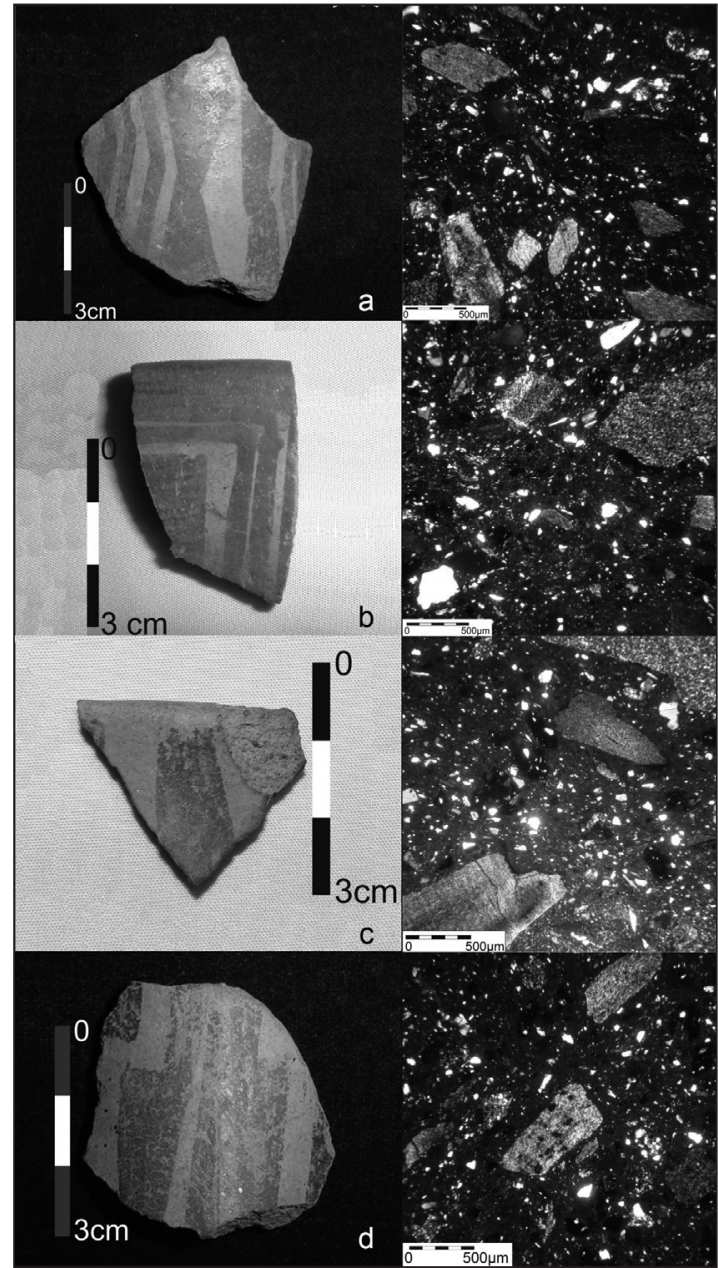

Figura 5. Fotos de fragmentos y microfotografías de pastas de la cerámica Vaquerías: a. Muestra 1; b. Muestra 2; c. Muestra 3; d. Muestra 4.

ro. Como se observa en la Tabla 3 y en las Figuras 5 y 6 , predomina la estructura de fondo criptofilitosa, aunque en algunos ejemplos se presenta algo microgranosa, registrándose microcristales de cuarzo. Además se reconocieron microlaminillas de biotita $\left(\mathrm{M}_{2}\right)$ y microcristales de plagioclasas $\left(\mathrm{M}_{4}\right)$.

El análisis petrográfico determinó que las pastas analizadas tienen entre un 18 y $26 \%$ de inclusiones mayores a $15 \mu \mathrm{m}$. Se reconocieron en todos los casos litoclastos metamórficos (pizarra-filita), arenisca, cristaloclastos de cuarzo y tiesto molido. Además, de forma escasa, se registraron feldespato potásico, plagioclasas, microcli-

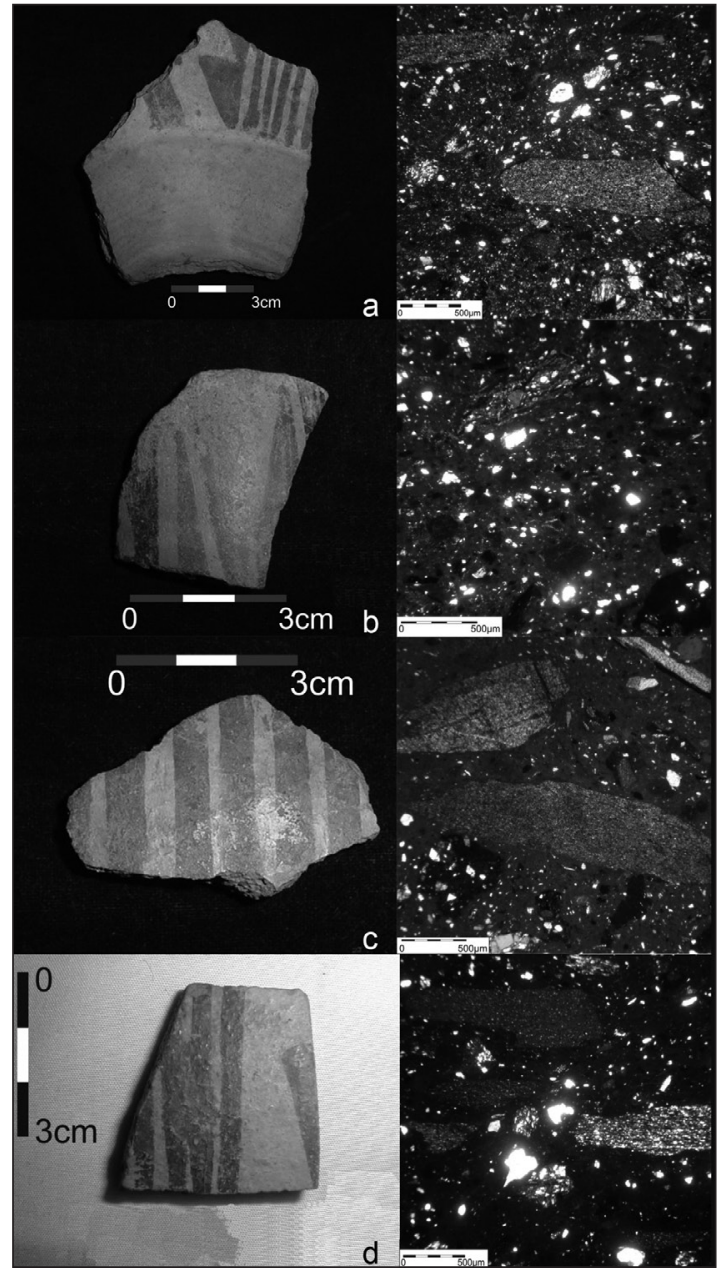

Figura 6. Fotos de fragmentos y microfotografías de pastas de la cerámica Vaquerías: a. Muestra 5; b. Muestra 6; c. Muestra 7; d. Muestra 8.

no, biotita, muscovita, anfíboles-piroxenos, carbonatos, minerales opacos, vidrio volcánico y gránulos de arcilla (Tabla 4). Los litoclastos metamórficos presentan formas tabulares. Los cristaloclastos de cuarzo son subangulares, subredondeados y redondeados. Los tiestos molidos tienen formas irregulares. Aunque la granulometría de las inclusiones oscila entre el limo grueso y la arena muy gruesa $(30 \mu \mathrm{m}$ a $2000 \mu \mathrm{m})$, la mayoría de los atemperantes se presentan como una arena gruesa $(500 \mu \mathrm{m}$ a $1000 \mu \mathrm{m})$. Las cavidades son escasas, representan un $2 \%$ del total de la pasta pero en algunos casos este porcentaje es mayor alcanzando hasta 5,60\% (Mi). En general, tienen formas alargadas e irregulares pero también 


\begin{tabular}{|c|c|c|c|c|c|c|c|c|}
\hline M & $\mathbf{1}$ & $\mathbf{2}$ & $\mathbf{3}$ & $\mathbf{4}$ & $\mathbf{5}$ & $\mathbf{6}$ & $\mathbf{7}$ & $\mathbf{8}$ \\
\hline Mat & $69,60 \%$ & $75,68 \%$ & $72,26 \%$ & $75,66 \%$ & $72,08 \%$ & $80,54 \%$ & $70,58 \%$ & $74,19 \%$ \\
\hline Cav & $5,60 \%$ & $2,90 \%$ & $2,40 \%$ & $2,22 \%$ & $5,33 \%$ & $1,95 \%$ & $3,12 \%$ & $1,61 \%$ \\
\hline Qz & $6,20 \%$ & $8,30 \%$ & $5,48 \%$ & $6,92 \%$ & $5,10 \%$ & $5,84 \%$ & $5,46 \%$ & $4,16 \%$ \\
\hline FK & $0,00 \%$ & $0,19 \%$ & $0,34 \%$ & $0,00 \%$ & $0,00 \%$ & $0,00 \%$ & $0,00 \%$ & $0,00 \%$ \\
\hline Plag & $0,60 \%$ & $0,58 \%$ & $0,34 \%$ & $0,27 \%$ & $0,22 \%$ & $0,32 \%$ & $0,19 \%$ & $0,23 \%$ \\
\hline Mic & $0,00 \%$ & $0,00 \%$ & $0,00 \%$ & $0,00 \%$ & $0,00 \%$ & $0,00 \%$ & $0,10 \%$ & $0,00 \%$ \\
\hline B & $0,00 \%$ & $0,19 \%$ & $0,34 \%$ & $0,00 \%$ & $0,22 \%$ & $0,00 \%$ & $0,39 \%$ & $0,00 \%$ \\
\hline M & $0,20 \%$ & $0,19 \%$ & $0,00 \%$ & $0,00 \%$ & $0,22 \%$ & $0,32 \%$ & $0,19 \%$ & $0,23 \%$ \\
\hline C & $0,20 \%$ & $0,00 \%$ & $0,00 \%$ & $0,00 \%$ & $0,00 \%$ & $0,00 \%$ & $0,00 \%$ & $0,00 \%$ \\
\hline A-P & $0,20 \%$ & $0,00 \%$ & $0,34 \%$ & $0,00 \%$ & $0,00 \%$ & $0,00 \%$ & $0,10 \%$ & $0,00 \%$ \\
\hline A & $2,00 \%$ & $0,97 \%$ & $2,40 \%$ & $2,49 \%$ & $3,19 \%$ & $0,97 \%$ & $2,53 \%$ & $2,53 \%$ \\
\hline P-F & $12,80 \%$ & $7,92 \%$ & $11,99 \%$ & $6,37 \%$ & $10,70 \%$ & $6,17 \%$ & $7,80 \%$ & $11,29 \%$ \\
\hline VV & $0,60 \%$ & $0,00 \%$ & $0,68 \%$ & $0,27 \%$ & $0,45 \%$ & $0,32 \%$ & $0,19 \%$ & $0,00 \%$ \\
\hline G & $0,40 \%$ & $2,08 \%$ & $0,34 \%$ & $1,38 \%$ & $0,22 \%$ & $0,97 \%$ & $0,39 \%$ & $0,00 \%$ \\
\hline TM & $1,20 \%$ & $1,00 \%$ & $2,74 \%$ & $4,15 \%$ & $1,59 \%$ & $2,60 \%$ & $8,77 \%$ & $5,53 \%$ \\
\hline MO & $0,40 \%$ & $0,00 \%$ & $0,34 \%$ & $0,27 \%$ & $0,68 \%$ & $0,00 \%$ & $0,19 \%$ & $0,23 \%$ \\
\hline
\end{tabular}

Tabla 4. Porcentajes de matriz, cavidades y material no plástico observados en los cortes delgados. Mat: matriz; Cav: cavidades; Qz: cuarzo; FK: feldespato potásico; Plag: plagioclasas; Mic: microclino; B: biotita; M: muscovita; C: carbonatos; A-P: anfíboles-piroxenos; A: arenisca; P-F: pizarra-filita; VV: vidrio volcánico; G: gránulos de arcilla; TM: tiesto molido; MO: minerales opacos.

se registraron algunas cavidades redondeadas (M8). Por último, las cavidades tienen tamaños que varían entre $400 \mu \mathrm{m}$ y $500 \mu \mathrm{m}$.

A partir de estas observaciones, se pueden inferir algunas pautas técnicas recurrentes. En primer lugar, se postula que las alfareras $y / 0$ alfareros elaboraron estas vasijas usando arcillas con escasas inclusiones naturales a las cuales incorporaron atemperantes gruesos. En segundo lugar, se observa una matriz compacta sin microlaminillas de micas (biotita y/o muscovita) y donde las inclusiones se encuentran bien cementadas. Esta cementación de los atemperantes y la escasez de cavidades estarían indicando un trabajo de preparación de la pasta cerámica con un buen amasado de la misma. En tercer lugar, se puede asumir el empleo de una cocción controlada, dada la homogeneidad de la estructura de fondo de la matriz en todo el corte delgado. Por último, la estructura criptofilitosa se podría relacionar con temperaturas de cocción de alrededor de $800^{\circ} \mathrm{C}$ (ver Cremonte 1996: 202).

Como se ha mencionado más arriba, solo se encuentra publicada la información del análisis petrográfico efectuado a un tiesto Vaquerías procedente del sitio Río Las
Piedras, valle de Tafí (Cremonte 1996). La pasta de este fragmento presenta un color rojo intenso que contrasta con el color de las inclusiones. Las mismas son cristaloclastos de cuarzo, biotita, litoclastos de cuarcita y pizarras angulosas, estas últimas las más abundantes. La granulometría predominante es arena y arena gruesa, y las cavidades son grandes. Además es importante resaltar que en una etapa comparativa se determinó que esta pasta se asemeja composicionalmente a las pastas de los fragmentos Vaquerías provenientes de Quebrada del Toro (ver Cremonte 1996: 128-131 y 269-270).

Esta descripción permite observar fuertes similitudes entre los materiales estudiados por Cremonte y las cerámicas aquí analizadas de Cardonal, Bordo Marcial y Tolombón.

\section{* Vaquerías en las aldeas cardonal y BORDO MARCIAL DEL VALLE DEL CAJÓN}

A pesar de la amplia dispersión reconocida, en pocos casos la cerámica Vaquerías fue hallada en sitios arqueológicos con excavaciones y estratigrafías bien analizadas. 


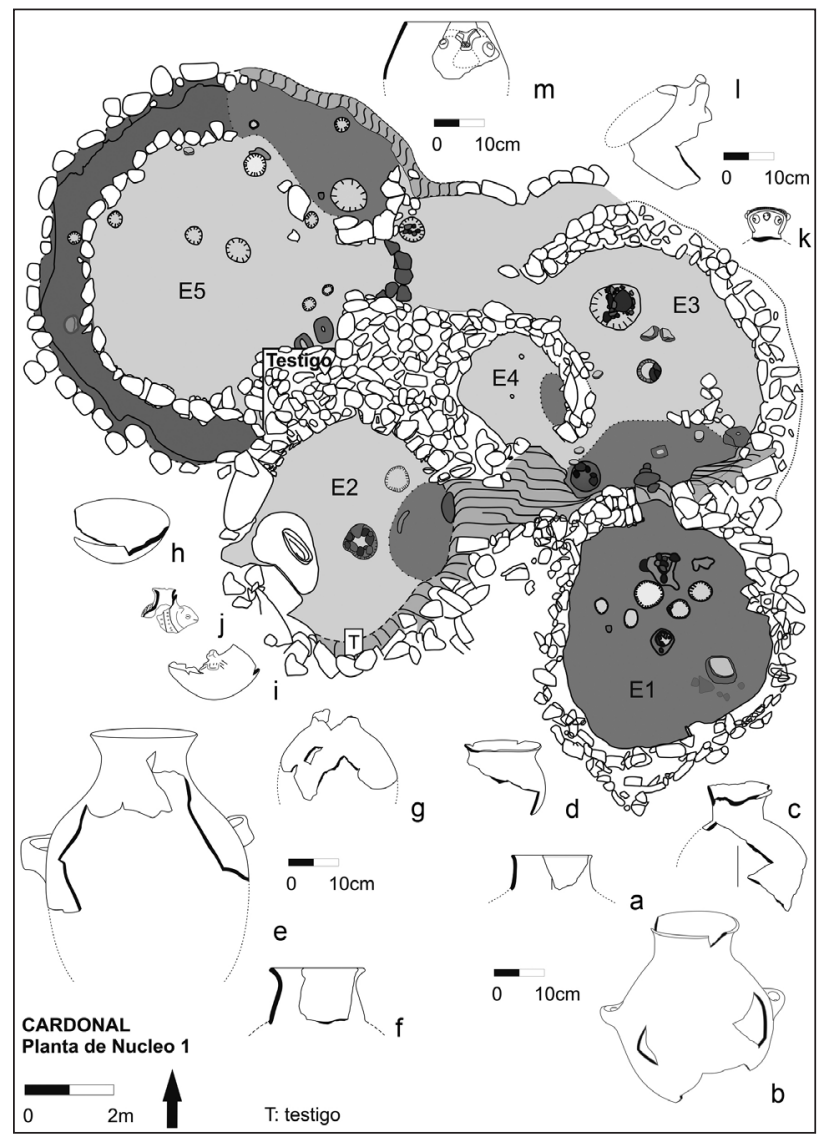

Figura 7. Recipientes hallados en el Núcleo 1 de Cardonal. a. Olla ordinaria; b. Vasija subglobular con dos asas; c. Cántaro un baño rojo; d. Olla globular pequeña beige pulido; e. Olla ordinaria; f. Olla ordinaria; g. Cántaro beige pulido; h. Cuenco hemisférico gris pulido; i. Vasija globular con aplique modelado; j. Vasija efigie con motivo zoomorfo; k. Vasija con modelado zooantropomorfo; 1. Olla ordinaria calceiforme; $\mathbf{m}$. Olla con representación antropomorfa al pastillaje.

En menor medida, estos materiales fueron recuperados en contextos claramente domésticos. Por lo tanto, nuestros hallazgos de este material en las aldeas de Cardonal y Bordo Marcial resultan muy significativos, ya que aparecen en contextos bien preservados, como parte del piso de ocupación de viviendas bien estudiadas y con fechados radiocarbónicos.

En la aldea de Cardonal se excavó en forma completa un núcleo habitacional con cinco recintos. En esta vivienda se recuperaron enseres de cocina, almacenamiento y consumo de alimentos, una pipa de cerámica para fumar, puntas de proyectil, improntas de cestería y gran cantidad de artefactos de molienda. Además, se registraron entre otros rasgos arquitectónicos, hoyos de poste, un fo- gón en trípode y otras áreas de combustión que muestran un claro contexto doméstico. La ocupación se extiende entre $1932 \pm 35$ y $1781 \pm 35$ años radiocarbónicos AP y refleja la presencia del estilo Vaquerías integrando la vida cotidiana de esta aldea del primer siglo DC (Scattolin et al. 2009a, 2009b).

Esta cerámica aparece en la casa asociada a otros estilos, formas e iconografías reconocidos para el período Formativo, tales como representaciones figurativas modeladas y al pastillaje como las de Tafí, diseños geométricos incisos como en San Francisco y Río Diablo, representaciones zoomorfas similares a las presentes en Candelaria y recipientes Grises Pulidos de pastas finas (Figura 7). 


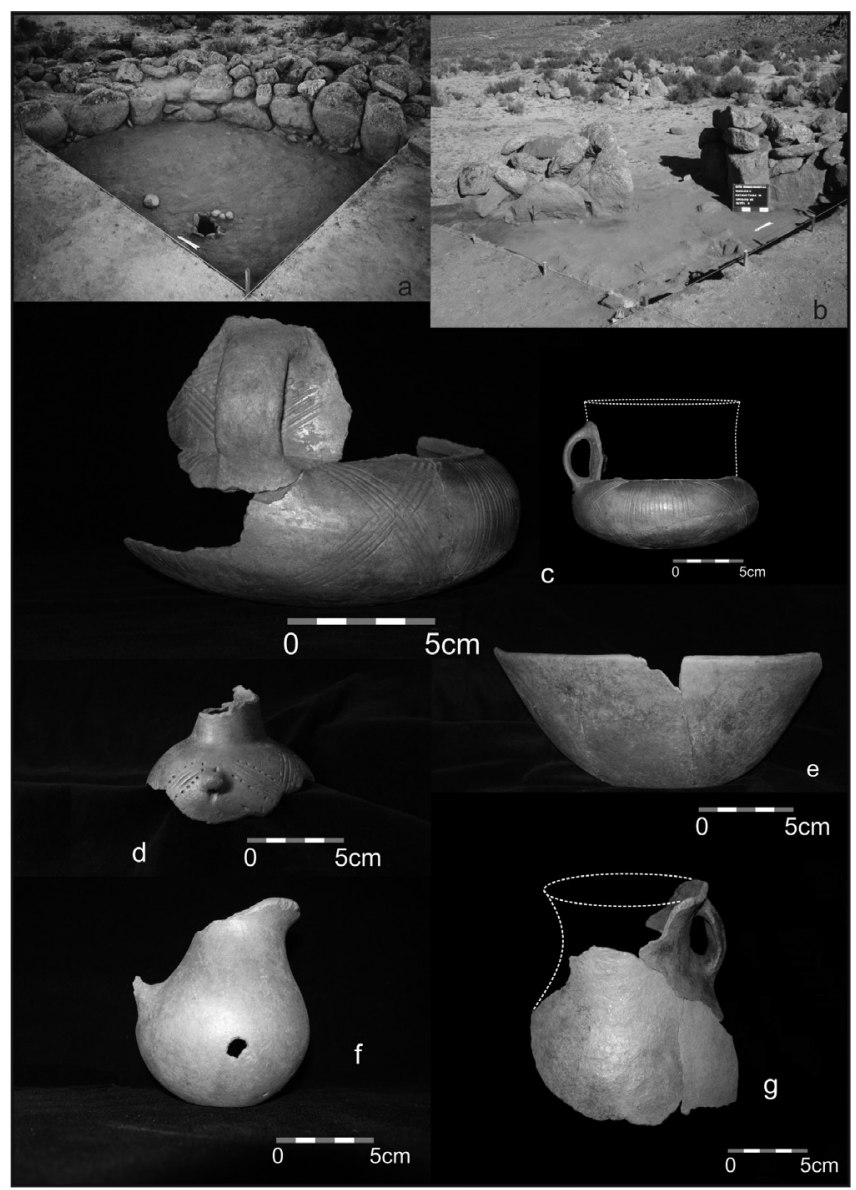

Figura 8. Bordo Marcial. a. Estructura 18 Cuadrante NE; b. Estructura 18 Cuadrante SE; c. Jarra pequeña de perfil compuesto Río Diablo; d. Vasija de cuello restringido con diseños incisos y pastillaje; e. Cuenco gris pulido; f. Vasija globular pequeña rojo pulido; g. Vasija mediana con dos asas verticales en cinta.

En un trabajo previo sobre el material cerámico del sitio Cardonal, se pudo determinar la presencia de cuatro tipos diferentes de pastas cerámicas además del Tipo Vaquerías. Un primer grupo (Cardonal Tipo I) está constituido por pastas gruesas con $35 \%$ de inclusiones, representadas en su mayoría por litoclastos graníticos y cristaloclastos de cuarzo. Las granulometrías registradas son principalmente de arena gruesa y arena muy gruesa $(500 \mu \mathrm{m}$ a $2000 \mu \mathrm{m})$. Un segundo tipo (Cardonal Tipo II) presenta una pasta con $28 \%$ de inclusiones, que en su mayoría corresponden a litoclastos graníticos alterados y seudomorfos de muscovita. La granulometría observada varía entre arena y arena muy gruesa $(250 \mu \mathrm{m}$ a 2000 $\mu \mathrm{m})$. El tercer grupo (Cardonal Tipo III) responde a pastas cuyas inclusiones representan el 25\% de la misma, en su mayoría litoclastos graníticos y cristaloclastos de cuarzo. La granulometría observada es arena $(250 \mu \mathrm{m}$ a $500 \mu \mathrm{m})$. Por último, el cuarto grupo determinado hasta el momento (Cardonal Tipo IV) está conformado por pastas donde los elementos no plásticos representan hasta el 8\%, tratándose posiblemente de inclusiones naturales de las arcillas. Las similitudes de las características petrográficas de los grupos Cardonal Tipo I, Tipo III y Tipo IV con la litología circundante a la aldea permitieron postular que las cerámicas fueron producidas de forma local. En cambio, por las características petrográficas de las pastas Tipo Vaquerías descriptas más arriba -y que aglomera solamente los fragmentos del estilo Vaqueríasse puede postular que esta cerámica no se produjo al sur de los valles Calchaquíes, es decir, se presenta como una 
manufactura alóctona al área de estudio (Pereyra Domingorena 2010).

Este material fue encontrado en cuatro de los cinco recintos. En la Estructura E1 donde se constataron actividades de cocina, los fragmentos Vaquerías están asociados a una olla globular grande con restos de hollín, otra vasija beige con dos asas y terminación pulida, un cántaro con baño rojo y una pequeña olla globular beige pulida en líneas (Figuras 7a, b, c y d). En la Estructura E2, se halla junto a dos grandes ollas de cocina o almacenamiento con cuellos restringidos de pastas Cardonal Tipo I (Figura 7 e y f), un cántaro beige pulido de pasta Cardonal Tipo III (Figura 7g) y tres recipientes con pastas Cardonal Tipo IV representadas por un cuenco hemisférico pulido, una vasija de cuerpo globular con un pequeño aplique modelado cerca de su diámetro máximo y una vasija efigie zoomorfa confeccionada usando la incisión y el pastillaje (Figura 7 h, i y j).

En la estructura $E_{3}$, la cerámica se asocia con un recipiente con modelado zooantropomorfo de pasta Cardonal Tipo II (Figura $7 \mathrm{k}$ ) y una olla ordinaria calceiforme de pasta Cardonal Tipo I (Figura 7l). Por último, en la estructura E5 aparece material Vaquerías junto a una olla con la representación de un rostro antropomorfo al pastillaje (Figura $7 \mathrm{~m}$ ).

En la aldea de Bordo Marcial, se excavó en forma completa una estructura (Figuras $8 \mathrm{a}$ y b) que fue fechada en $1869 \pm 38$ años radiocarbónicos AP (Pereyra Domingorena 2010). En este recinto, los fragmentos Vaquerías se encuentran asociados a cinco recipientes. Uno de ellos es una jarra pequeña de perfil compuesto, con cuerpo elipsoide, cuello cilíndrico y motivos incisos de puntos y rombos asimilable a las conocidas como Río Diablo (Figura 8c). Otra vasija presenta un cuello muy restringido con diseños incisos y con un pastillaje (Figura $8 d$ ). Un cuenco gris pulido (Figura 8e), una vasija globular muy pequeña rojo pulido (Figura $8 f$ ) y otra vasija mediana con dos asas verticales en cinta (Figura 8g) completan el conjunto de vasijas recuperado en esta habitación.

Como puede observarse, la alfarería Vaquerías está presente en las dos aldeas, ocupando distintos lugares de la vivienda. Un aspecto interesante, que retomaremos en las discusiones, es que si bien pudieron reconocerse algunas de las formas de estas piezas y sus diseños, el material Vaquerías aparece muy fragmentado y no hubo posibilidades de remontaje.

\section{* El contexto cronológico y LAS ASOCIACIONES ESTILÍSTICAS}

En un primer momento, el estilo Vaquerías fue ubicado cronológicamente entre el 200 y el 400 DC (Heredia et al. 1974a), basándose en asociaciones estilísticas. Esta consideración fue reforzada por los fechados obtenidos por Cigliano y su equipo en Las Cuevas, Quebrada del Toro, lugar donde el material tricolor apareció en estratigrafía. Estos investigadores consideraron “...una antigüedad mayor para esta tradición policroma, la cual podría remontarse, promediando las dos fechas de Las Cuevas, al 200 D.C., aproximadamente" (Cigliano et al. 1972: 233).

Posteriormente, otro fechado del mismo sitio también se asoció al material Vaquerías. Este fechado de $2150 \pm 80$ años radiocarbónicos AP proviene del Montículo Sur (LC E1o $\mathrm{C}_{4}$ ) y corresponde a la capa inmediatamente inferior a la que se encontró este estilo (Cigliano et al. 1976). Los sitios Potrero Grande y Cerro El Dique, donde del mismo modo aparece Vaquerías, presentan "...fechas radiocarbónicas de $240 \pm 50$ años DC [...] y 260 $\pm 50 . .$. " (Cigliano et al. 1976: 123), respectivamente.

En el sitio El Mollar, ubicado en el valle de Tafí también se han encontrado fragmentos de este estilo. La excavación de la trinchera realizada en el montículo presenta varios fechados que sitúan el componente Formativo entre los $1955 \pm 55$ y $1880 \pm 65$ años radiocarbónicos AP (Núñez Regueiro y Azcárate 1996).

Datos publicados más recientemente permiten ajustar esta temporalidad y suman evidencias cronológicas de otras zonas como los valles mesotermales. Un fechado del sitio Soria 2, obtenido de los carbones de un fogón en un contexto doméstico en el sur del valle de Santa María, donde también se encontró un fragmento Vaquerías, ubica este estilo en $1940 \pm 80$ años radiocarbónicos AP (Palamarczuk et al. 2007). A estos fechados debemos adicionar las seis dataciones con que se cuenta para el área de La Quebrada mencionadas más arriba. 
Distante de estos valles, se encuentra la aldea de Tulor I en el oasis de San Pedro de Atacama, en la cual se hallaron tres fragmentos Vaquerías. Estos materiales fueron hallados en el piso inferior de una de las habitaciones el cual tiene un fechado cuyo rango cronológico es de 200245 DC (Llagostera et al. 1984).

Considerando los fechados con que se cuenta actualmente, podemos esbozar para la cerámica Vaquerías una cronología más extensa, que se iniciaría en los últimos siglos AC y finalizaría en el siglo IV DC. Es interesante notar que los fechados más extremos de este rango provienen de sitios de quebrada del Toro.

Por otra parte, en Bordo Marcial las asociaciones estilísticas entre Vaquerías y piezas de formas y diseños de la fase Río Diablo (González 1977) marcan una temporalidad dentro del rango estimado anteriormente.

Esta presencia de materiales Vaquerías junto a otros de la fase Río Diablo también fue advertida en el valle de Hualfín y mencionada por González y Cowgill (1975), cuando presentan la cronología para este valle. Posteriormente, en una de las tumbas excavadas por Berberián y Massidda (1975) en el sitio Las Barrancas (tumba 2) esta asociación se repite. En esta tumba se hallaron, entre otras piezas, dos vasijas Vaquerías de cuerpo esférico y cuello cilíndrico muy largo y ligeramente evertido, otra escudilla del mismo estilo con motivos zoomorfos (batracios) pintados y modelados y dos jarritos con cuerpo globular algo achatado y cuello cilíndrico con representaciones incisas de rombos, puntos y líneas pertenecientes a Río Diablo (Berberián y Massidda 1975: lams. II, VI y IX). En la tumba 1 del mismo sitio, una pieza Vaquerías de contorno inflexionado, con diseños geométricos en rojo y la representación también pintada de un rostro antropomorfo se halló junto con una escudilla roja pulida con un pequeño modelado aplicado en el borde, una escudilla gris con diseños incisos y dos piezas de pasta ordinaria, un vaso y una vasija subglobular (Berberián y Massidda 1975: lams. I y III).

También en tumbas de quebrada del Toro, se conocen asociaciones contextuales entre materiales Vaquerías y otros conjuntos alfareros. El ajuar de la Tumba 3 del sitio Cerro El Dique contenía dos piezas tricolores Vaquerías (Cigliano et al. 1972: figs. 1 y 3 ) junto a cerámica gris pu- lida, rojo pulida y corrugada. En la tumba 1 de Potrero Grande se halló una particular pieza Vaquerías que representa un ofidio (Cigliano et al. 1972: fig. 2).

Por su parte, en las yungas orientales, durante la fase Chuscha, aparecen materiales Vaquerías asociados con tipos cerámicos Candelaria incisos, grabados y pintados (Heredia 1974a), mientras que en el valle de Tafí, en el sitio el Mollar, se encuentra Vaquerías junto a materiales de la tradición Tafí y también con fragmentos cerámicos de estrecha similitud con alfarería de la tradición San Francisco (Tartusi y Núñez Regueiro 1993). Esta última asociación se repite en el valle de Lerma (González 1983; Escobar 2008).

Por otra parte, hay que señalar que se han planteado afinidades estilísticas entre el conjunto Vaquerías y la cerámica San Francisco, sobre todo en relación a los elementos del diseño (Dougherty 1977; González 1998; Ortiz 2007). Son llamativas las similitudes entre los diseños de Vaquerías y algunos elementos geométricos hallados en los tipos cerámicos Incisos y Pintados Rojo sobre Ante del valle de San Francisco entre los que se destacan los escalonados, banderines y rombos. De hecho, estas afinidades han llevado a plantear que el origen del estilo Vaquerías podría encontrarse en la cerámica bicolor del Complejo San Francisco, mientras que también se han destacado las similitudes entre diseños incisos que se manifiestan tanto en San Francisco como en Río Diablo (Dougherty 1977).

\section{* Consideraciones finales}

A través de nuestros estudios, se conocieron características y particularidades del material Vaquerías que se presenta como un conjunto homogéneo. Las pastas manifestaron regularidades en los modos técnicos empleados para su manufactura. Además, se advirtió el uso de un surtido de diseños geométricos pintados que, aunque combinados y recombinados, responden a un repertorio poco extenso.

Una de las características observadas a ojo desnudo y en fractura fresca es el color beige grisáceo de sus pastas y la presencia de inclusiones negras, marrones oscuras y rojizas. Estas inclusiones se hallan bien cementadas en la matriz arcillosa, lo que nos permiten pensar en una tradición artesanal que implicó un buen trabajo de amasado 
y la búsqueda de altas temperaturas a fin de lograr pastas compactas y duras, que no se desgranan a pesar del porcentaje elevado del atemperante. Otro de los rasgos visibles es el tratamiento de la superficie de las piezas, donde se observa un engobe uniforme y de terminación bien lograda cuyo color puede variar entre el blanco amarillento y el anaranjado claro. A través del análisis petrográfico, hemos podido reconocer que se usaron arcillas a las cuales se les agregaron inclusiones de tipo pizarrafilita, cuarzo, arenisca y tiesto molido, en proporciones que varían entre $20 \%$ y $25 \%$ del total de la pasta y con granulometría de arena y arena gruesa. Además se destacó la escasez de cavidades.

Los diseños geométricos pintados responden a un acotado número de elementos sólidos y de línea que se combinan en múltiples configuraciones de acuerdo a arreglos de simetría y donde el color también es un elemento clave. Algunos ejemplares excepcionales corresponden a vasijas 'efigie' antropomorfas y zoomorfas, donde además no dejan de estar presentes los diseños geométricos característicos del estilo Vaquerías. Si bien en algunos ejemplares puede percibirse menor pericia en el trazo de la línea pintada o en la terminación del diseño, se advierte una búsqueda común en lo que hace al efecto final de la pieza, que es resultado de reglas compartidas en el hacer, elegir figuras, seleccionar colores y arreglos de simetría.

Los resultados aquí presentados y las comparaciones realizadas con los escasos datos publicados de otras áreas del NOA, permiten señalar la unidad de la cerámica Vaquerías, tanto en lo referente a los diseños y las representaciones plásticas como en la composición de sus pastas.

En otras oportunidades, se han sugerido como posibles áreas de manufactura de piezas Vaquerías el valle de Lerma, el valle de Hualfín (Korstanje 1995) y la quebrada del Toro (Cremonte 1996). Los materiales aquí estudiados no nos permiten sostener o corroborar ninguna de estas hipótesis. Solo un estudio que abarque los materiales locales y alóctonos de cada una de estas áreas, permitirá avanzar sobre esta cuestión. Sin embargo, es posible fundamentar el carácter alóctono de los materiales Vaquerías en el valle del Cajón a partir de su comparación con las diferentes pastas registradas en el material cerámico del sitio Cardonal, cuyo posible carácter local fue señalado anteriormente (Pereyra Domingorena 2009, 2010). En algunas de estas últimas pastas se hallaron litoclastos graníticos migmatíticos y cristaloclastos de cuarzo con extinción ondulante característicos de un ambiente geológico de metamorfismo de alto grado. Tal fenómeno es propio de la sierra de Chango Real, a cuyos pies se encuentran las aldeas de Cardonal y Bordo Marcial. Esta serranía es parte de la Formación Chango Real, un macizo del Terciario constituido por migmatitas graníticas (Turner 1973). Por lo tanto, la litología circundante a los sitios es coincidente con los atemperantes usados en la preparación de las pastas cerámicas. Esta relación permite sustentar la hipótesis de la producción local para gran parte de la alfarería hallada en Cardonal.

Por su parte, en el material Vaquerías los elementos graníticos migmatíticos están ausentes. Las inclusiones no plásticas propias de esta cerámica (litoclastos metamórficos) no han sido registradas en la geología del área de estudio. Como en esta zona no se han detectado formaciones con pizarras-filitas, es muy posible que el o las áreas de producción de esta cerámica se encuentren en ambientes metamórficos de bajo grado ubicados en otras regiones. Por estas razones y a modo de hipótesis, se considera a la alfarería Vaquerías como material foráneo obtenida a través de las redes activas de circulación e interacción regional de bienes (Scattolin y Lazzari 1997).

Tartusi y Núñez Regueiro (1993) sostuvieron que la cerámica Vaquerías constituye un indicador del intercambio de objetos e ideas a través del movimiento de grupos caravaneros dedicados a la distribución de bienes de carácter ritual. Por su parte, Lazzari (2005) argumenta que Vaquerías, así como otros estilos cerámicos y otras materialidades, constituye parte de redes de intercambio mucho más complejas y flexibles donde distintos universos estilísticos podrían estar insertos en una trama de interacción social. En Cardonal y Bordo Marcial, esta interacción y circulación se manifiesta a partir de la presencia de distintos objetos dentro de los mismos contextos domésticos: obsidianas de la Puna catamarqueña, cerámica Vaquerías y otras con diseños que remiten a las selvas orientales (Scattolin et al. 2009a) y evidencia de consumo de cebil (Bugliani et al. 2010).

Por otra parte, se ha iniciado un programa de análisis de activación neutrónica instrumental (AANI) para la identificación de huellas químicas propias de las arcillas usadas 
en la producción alfarera. De esta manera, se espera dilucidar posibles áreas de procedencia de la alfarería estudiada (Lazzari et al. 2009). La integración futura de la información estilística, morfológica, petrográfica y química de la cerámica Vaquerías permitirá avanzar sobre la determinación del área de manufactura de esta cerámica polícroma.

Por otra parte, en relación a la presencia y consumo de bienes cerámicos en los contextos domésticos de Cardonal y Bordo Marcial, es notable la diferencia de integridad entre los recipientes cerámicos de diferentes tipos y estilos. Así, algunos se han conservado parcialmente enteros $\mathrm{o}$, aunque fragmentados, pudieron ser reconstruidos. Por el contrario, los materiales Vaquerías se encuentran representados por fragmentos que no pueden ser remontados como piezas enteras. Esta situación nos hace pensar que los fragmentos Vaquerías podrían haber participado de las prácticas de la vida cotidiana en La Quebrada, no solo como recipientes sino también manteniendo o recreando su valor una vez fragmentados. En este sentido, estas cerámicas alóctonas podrían, como han planteado otros autores para otros contextos, ser sostenedores de narrativas (Haber 2007), fragmentos que han sido inten- cionalmente conservados, posiblemente como referentes de historias de relaciones, intercambios e interacción.

Finalmente, creemos que la profundización de estas diferentes líneas de investigación permitirá ampliar el conocimiento sobre Vaquerías, evaluando no solo sus características formales y de manufactura, sino también ahondando en el entramado social del cual fue parte.

Agradecimientos A la Dra. T. Montenegro (Cátedra de Mineralogía FCEyN - UBA), a la Dra. A. Díaz (IGyMUNJu) y a la Dra. B. Cremonte (IGyM-UNJu) por la ayuda brindada para mejorar el registro de lo observado en los cortes delgados. A M. C. Scattolin, por compartir sus registros fotográficos de piezas Vaquerías. Al Museo Etnográfico, que posibilitó el análisis de material cerámico de sus colecciones. A las autoridades de la Municipalidad de San José por facilitarnos el traslado durante los trabajos de campo. A los habitantes de La Quebrada por su amena colaboración durante nuestra estadía en su localidad. Estas investigaciones fueron financiadas a través del ANPCyT-PICT 0420194 dirigido por M. C. Scattolin y el ANPCyT-PICT 0601244 dirigido por M. F. Bugliani.

\section{* Referencias citadas}

ADAMS, A. E., W. S. MACKENZIE y C. GUILFORD, 1997 [1984]. Atlas de rocas sedimentarias. Masson, Barcelona.

BERBERIÁN, E., y F. MASSIDDA, 1975. Investigaciones arqueológicas en Las Barrancas (Dpto. de Belén, Catamarca). Nuevas contribuciones para el estudio de la cultura Condorhuasi del Noroeste Argentino. Revista del Instituto de Antropología. Tercera Serie 2: 7-48.

BUGLIANI, M. F., C. M. CALO y M. C. SCATTOLIN, 2010. Fumando en la cocina. Determinación de contenidos por técnicas físicoquímicas en dos pipas cerámicas del sitio Cardonal. En La arqueometría en Argentina y Latinoamérica, S. Bertolino, R. Cattáneo y A. Izeta (Eds.), pp. 231-236. Editorial de la Facultad de Filosofía y Humanidades, Córdoba.

CASTRO, V., C. ALDUNATE, J. BERENGUER, L. CORNEJO, C. SINCLAIRE y V. VARELA, 1994. Relaciones entre el Noroeste Argentino y el Norte de Chile: el sitio O2-TU-002, Vegas de Turi. En De costa a selva: Producción e intercambio entre los pueblos agroalfareros de los Andes Centro-Sur, M. E. Albeck (Ed.), pp. 215236. Instituto Interdisciplinario Tilcara, Facultad de Filosofía y Letras, Universidad de Buenos Aires, Tilcara.

CIGLIANO, E., 1970. Problemas referentes al sitio arqueológico de Las Cuevas, Departamento de Rosario de Lerma, Provincia de Salta. Relaciones de la Sociedad Argentina de Antropología V: 99-104.

CIGLIANO, E., R. RAFFINO y H. CALANDRA, 1972. Nuevos aportes para el conocimiento de las entidades alfareras más tempranas del Noroeste Argentino. Relaciones de la Sociedad Argentina de Antropología VII: 225-236.

1976. La aldea formativa de las Cuevas (Provincia de Salta). Relaciones de la Sociedad Argentina de Antropología X: 73-130.

CREMONTE, M. B., 1996. Investigaciones arqueológicas en la Quebrada de la Ciénega. (Dpto. de Tafi, Tucumán). Tesis doctoral, Facultad de Ciencias Naturales y Museo, Universidad Nacional del La Plata, La Plata. 
CREMONTE, M. B., N. FLEGENHEIMER y L. DE SANTIS, 1987. El yacimiento de Las Garzas, Valle de Lerma, Salta. Boletín del Colegio de Graduados en Antropología 16: 21-28.

CURTOIS, L., 1976. Examen au microscope pétrographique des céramiques archéologiques. Notes et monographies techniques, 8. CNRS, Paris.

DE FEO, M. E., 2007. Revisando antiguas cuestiones: nuevas evidencias acerca de la cronología y organización del espacio en el sitio Tres Cruces (Quebrada del Toro, Salta). Cuadernos FHyCS, UNJu 32: 91-109.

DOUGHERTY, B., 1977. Análisis de la variación en el complejo San Francisco. Obra del Centenario del Museo de La Plata, tomo II: 237252.

ESCOBAR, J. M., 1996. El período agroalfarero temprano en el valle de Lerma: El caso del sitio Silisque-Tilián 2. (Depto. de Chicoana, Pcia. de Salta). Actas y memorias del XI Congreso Nacional de Arqueología Argentina (II ${ }^{a}$ parte). Revista del Museo de Historia Natural de San Rafael XXIII (1/4): 33-49.

2008. Período Formativo Inferior del valle de Salta (Salta Argentina) Una interpretación. La Terminal Gráfica, La Plata.

FERNÁNDEZ DISTEL, A., 1998. Arqueología del formativo en la puna jujeña (1800 AC al 650 DC). Centro Argentino de Etnología Americana, Buenos Aires.

GONZÁLEZ, A. R., 1956. La cultura Condorhuasi del Noroeste Argentino (apuntes preliminares para su estudio). Runa VII: $37-85$

1977. El arte precolombino de la Argentina. Introducción a su historia cultural. Filmediciones Valero, Buenos Aires

1983. Nota sobre religión y culto en el Noroeste Argentino prehispánico. A propósito de unas figuras antropomorfas del $\mathrm{Mu}-$ seo de Berlín. Baessler-Archiv Neue Folge XXXI: 219-282.

1998. Arte precolombino. Cultura La Aguada. Arqueología y diseños. Filmediciones Valero, Buenos Aires.

GONZÁLEZ, A. R., y M. BALDINI, 1989. Vaquerías: la más antigua alfarería polícroma del Noroeste Argentino. Más allá del objeto 14: 8-12.

GONZÁLEZ, A. R., y G. COWGILL, 1975. Cronología arqueológica del valle de Hualfín, Pcia. de Catamarca, Argentina. Obtenida mediante el uso de computadoras. Actas y trabajos del primer Congreso de Arqueología Argentina: 383-404.
GRAMAJO BÜHLER, C. M., 2009. Primera caracterización del conjunto cerámico de la Quebrada de Los Corrales (El Infiernillo, Tucumán). Serie Monográfica y Didáctica 48: 121-123.

HABER A., 1996. El temprano en perspectiva investigaciones arqueológicas en Tebenquiche. Actas y memorias del XI Congreso Nacional de Arqueología Argentina (11 a parte). Revista del Museo de Historia Natural de San Rafael XXIII (1/4): 77-81.

2007. Comentarios marginales. En Sociedades precolombinas surandinas. Temporalidad, interacción y dinámica cultural del NOA en el ámbito de los Andes Centro-Sur, V. Williams, B. Ventura, A. Callegari y H. Yacobaccio (Eds.), pp. 59-72. Instituto de Arqueología FFyL - Universidad de Buenos Aires, Buenos Aires.

HEREDIA, O., 1974. Investigaciones arqueológicas en el sector meridional de las Selvas Occidentales. Revista del Instituto de Antropología V: 73-132.

HEREDIA, O., y J. A. PÉREZ, 1972. Una nueva entidad cerámica del Noroeste Argentino. Diario La Prensa, 27 de agosto, Buenos Aires.

HEREDIA, O., J. A. PÉREZ y A. R. GONZÁLEZ, 1974a. La antigüedad de la cerámica polícroma en el Noroeste Argentino. Revista del Instituto de Antropología V: 133-151.

HEREDIA, O.; M. PALACIOS, A. LUZZI y L. NAUDEAU, 1974b Ms. Ensayo de un cuadro cronológico del sector meridional del Valle Calchaquí. Ponencia al III Congreso Nacional de Arqueología Argentina, Salta.

KERR, P. F., 1965. Mineralogía óptica. McGraw-Hill Book Company Inc., Nueva York.

KORSTANJE, M. A., 1995. Nuevas reflexiones en torno a Vaquerías, un estilo cerámico polémico. Cuadernos FHyCS, UNJu 5: 169-179.

1998. Desempolvando antigüedades: Consideraciones sobre el repertorio cerámico Vaquerías. Mundo de Antes 1: 69-117.

KRAPOVIKAS, P., 1977. Arqueología de Cerro Colorado (Departamento Yavi, Provincia de Jujuy, República Argentina). Obra del Centenario del Museo de La Plata, tomo II: 123-148.

MUNSELL COLOR, 2000. Munsell Soil color charts. GretagMacbeth, Nueva York.

1975. Munsell Soil color charts. GretagMacbeth, Nueva York.

LAZZARI, M., 2005. Travelling objects and spatial images: exchange relationships and the production of social space. En Global Archaeological Theory. Contextual Voices and Contemporary Thoughts, P. P. A. Funari, A. Zarankin y E. Stovel (Eds.), pp. 191-210. Kluwer, Nueva York. 
LAZZARI, M., L. PEREYRA DOMINGORENA, M. C. SCATTOLIN, L. CECIL, M. GLASCOCK y R. J. SPEAKMAN, 2009. Ancient social landscapes of northwestern Argentina: preliminary results of an integrated approach to obsidian and ceramic provenance. Journal of Archaeological Science 36: 1955-1964.

LLAGOSTERA, A., A. M. BERÓN P. y L. BRAVO V., 1984. Investigaciones arqueológicas en Tulor I. Estudios Atacameños 7: 133-151.

NÚÑEZ, L., V. ZLATER M. y P. NÚÑEZ H., 1975. Relaciones prehistóricas trasandinas entre el N.W. argentino y Norte chileno (Período Cerámico). Series Documentos de Trabajo 6: 1-24.

NÚÑEZ REGUEIRO, V., 1974. Conceptos instrumentales y marco teórico en relación al análisis del desarrollo cultural del Noroeste Argentino. Revista del Instituto de Antropología V: 169-190.

NÚÑEZ REGUEIRO, V., y J. GARCÍA AZCÁRATE, 1996. Investigaciones arqueológicas en El Mollar, Dpto. Tafí del Valle, Pcia. de Tucumán. Actas y memorias del XI Congreso Nacional de Arqueología Argentina (13 ${ }^{a}$ parte). Revista del Museo de Historia Natural de San Rafael XXV (1/2), pp. 87-97.

OLISZEWSKI, N., C. MATÍAS GRAMAJO BÜHLER, E. P. MAURI, G. E. MIGUEZ, A. C. MUNTANER y M. M. PANTORRILLA RIVAS, 2010. Caracterización de un entierro humano en la Quebrada de Los Corrales (El Infiernillo, Tucumán). Intersecciones en Antropología 11: 315-319.

ORTIZ, G., 2007. El paisaje macrorregional. Uso del espacio social expandido a través de la circulación de objetos. En Producción y circulación prehispánicas de bienes en el sur andino, A. E. Nielsen, M. C. Rivolta, V. Seldes, M. M. Vázquez y P. H. Mercolli (Comps.), pp. 305-328. Editorial Brujas, Córdoba.

PALAMARCZUK, V., R. SPANO, F. WEBER, D. MAGNÍFICO, S. LÓPEZ y M. MANASIEWICZ., 2007. Soria 2. Apuntes sobre un sitio Formativo en el valle de Yocavil (Catamarca, Argentina). Intersecciones en Antropología 8: 121-134.

PEREYRA DOMINGORENA, L., 2009. Análisis petrográfico de los recipientes cerámicos del sitio Cardonal. En Arqueometría Latinoamericana: Segundo Congreso Argentino y Primero Latinoamericano, vol. 1, O. M. Palacios, C. Vázquez, T. Palacios y E. Cabanillas (Eds.), pp. 40-46. Centro Atómico Constituyente - CNEA, San Martín.

2010. Manufacturas alfareras de las sociedades aldeanas del primer milenio DC al sur de los valles Calchaquíes. Tesis doctoral. Facultad de Filosofía y Letras, Universidad de Buenos Aires, Buenos Aires.

RAFFINO, R. A., G. RAVIÑA, L. BALDINI y A. IÁCONA, 1982. La expansión septentrional de la Cultura La Aguada en el N.O. argentino. Cuadernos del Instituto de Antropología y Pensamiento Latinoamericano 9: 179-82.
SANTONI, M, y E. BULIUBASICH, 1988. Dinámica de contacto cultural prehispánico entre Puna y Valles a través del estudio del sitio Cobres, Departamento La Poma, Salta. En Resúmenes del IX Congreso Nacional de Arqueología Argentina, pp. 28-29.

SCATTOLIN, M. C., 2000. Santa María durante el Primer Milenio A. D. ¿Tierra baldía? Arstryck 1995-1998: 63-83.

2006. Contornos y confines del universo iconográfico precalchaquí del valle de Santa María. Estudios Atacameños 32: 119-139.

SCATTOLIN, M. C., y M. LAZZARI, 1997. Tramando redes: obsidianas al oeste del Aconquija. Estudios Atacameños 14: 189-209.

SCATTOLIN, M. C.; L. PEREYRA DOMINGORENA, L. CORTÉS, M. F. BUGLIANI, C. M. CALO, A. D. IZETA y M. LAZZARI, 2007. Cardonal: una aldea formativa entre los territorios de valles y puna. Cuadernos FHyCS, UNJu 32: 211-225.

SCATTOLIN, M. C., M. F. BUGLIANI, L. I. CORTÉS, C. M. CALO, L. PEREYRA DOMINGORENA y A. D. IZETA, 2009a. Pequeños mundos: hábitat, maneras de hacer y afinidades en aldeas del valle del Cajón, Catamarca. Relaciones de la Sociedad Argentina de Antropología XXXIV: 251-274.

SCATTOLIN, M. C., L. CORTÉS, M. F. BUGLIANI, C. M. CALO, L. PEREYRA DOMINGORENA, A. D. IZETA y M. LAZZARI, 2009b. Built landscapes of everyday life: a house in an early agricultural village of northwestern Argentina. World Archaeology 41 (3): 396-414.

TARRAGÓ, M. N., 1989. Contribución al conocimiento arqueológico de las poblaciones de los oasis de San Pedro de Atacama en relación de los otros pueblos puneños, en especial, el sector septentrional del valle Calchaquí. Tesis doctoral, Facultad de Humanidades y Arte, Universidad Nacional de Rosario, Rosario.

TARRAGÓ, M. N., 1996. El Formativo en el Noroeste Argentino y el Alto Valle Calchaquí. Actas y memorias del XI Congreso Nacional de Arqueología Argentina (11 ${ }^{a}$ parte). Revista del Museo de Historia Natural de San Rafael XXIII (1/4): 103-119.

TARRAGÓ, M. N., y M. C. SCATTOLIN, 1999. La problemática del período Formativo en el valle de Santa María. Actas del XII Congreso Nacional de Arqueología Argentina. Tomo I: 142-153.

TARTUSI, M. R. A., y V .A. NÚÑEZ REGUEIRO, 1993. Los Centros Ceremoniales del NOA. Publicaciones del Instituto de Arqueología 5 (Serie: Ensayos 1): 1-49.

TURNER, J. C. M., 1973. Descripción geológica de la Hoja 11d, Laguna Blanca. Boletín 142. Servicio Nacional Minero Geológico, Buenos Aires. 